INFN-FE-14-93

Ferrara, December 1993

\title{
Future solar neutrino spectroscopy and neutrino properties
}

\author{
V Castellania), S Degl'Innocentib,c), G Fiorentinib,c), M Lissiad) and B Riccic,e)
}

\author{
a) Dipartimento di Fisica dell'Università di Pisa, I-56100 Pisa, Osservatorio Astronomico di Collurania I-64100 Teramo and Università \\ dell'Áquila I-67100 L'Aquila \\ b)Dipartimento di Fisica dellUniversità di Ferrara, I-44100 Ferrara \\ c) Istituto Nazionale di Fisica Nucleare, Sezione di Ferrara, I-44100 Ferrara \\ d)Dipartimento di Fisica dell'Università di Cagliari, I-09100 Cagliari and Istituto Nazionale di Fisica Nucleare, Sezione di Cagliari, \\ I-09100 Cagliari \\ e)Scuola di Dottorato dell'Università di Padova , I-35100 Padova
}

\begin{abstract}
The Gallex result, together with the constraints from the observed value of the solar constant, yields strong limits on the flux of neutrinos from the pp-I termination of the pp-chain, $62 \leq \Phi_{\mathrm{pp}+\mathrm{pep}}\left[10^{9} \mathrm{~cm}^{-2} \mathrm{~s}^{-1}\right] \leq 65$, provided that neutrinos are standard. This conclusion is essentially independent of solar models and it implies that the ppchain is shifted towards the pp-I termination, in comparison with the SSM predictions. As a consequence, we derive an upper bound on Berillium neutrinos: $\Phi_{\mathrm{Be}} \leq 3 \cdot 10^{9} \mathrm{~cm}^{-2} \mathrm{~s}^{-1}$, the violation of this bound being a clear signal of non-standard neutrino properties. We also find that, for a large class of solar models built so as to enhance the pp-I termination, the pep-neutrino flux is stable around $\Phi_{\text {pep }} \approx 1.5 \cdot 10^{8} \mathrm{~cm}^{-2} \mathrm{~s}^{-1}$. Detection of pep neutrinos will clearly discriminate standard neutrinos against MSW oscillations, this latter model giving $\Phi_{\text {pep }}{ }^{\mathrm{MSW}} \leq 3 \cdot 10^{7} \mathrm{~cm}^{-2} \mathrm{~s}^{-1}$.
\end{abstract}




\section{Introduction}

New generation experiments are being planned for the detection of monochromatic solar neutrinos, produced in electron capture $\left({ }^{7} \mathrm{Be}+\mathrm{e}^{-} \rightarrow{ }^{7} \mathrm{Li}+\mathrm{v}\right)$ and in the pep $\left(\mathrm{p}+\mathrm{e}^{-}+\mathrm{p} \rightarrow \mathrm{d}+\mathrm{v}\right)$ reactions $[1,2,3]$. In this letter we discuss what can be learnt from these future measurements about neutrino properties, independently as much as possible - of the Standard Solar Model (SSM).

Our discussion will be directly in terms of the main components of the solar neutrino flux: $\mathrm{pp}$ and pep-neutrinos from the pp-I termination (flux on Earth $\left.\Phi_{\mathrm{p}}=\Phi_{\mathrm{pp}}+\Phi_{\mathrm{pep}}\right) ;{ }^{7} \mathrm{Be}$-neutrinos from the pp-II termination $\left(\Phi_{\mathrm{Be}}\right)$; neutrinos from the $\mathrm{CNO}$ cycle $\left(\Phi_{\mathrm{CNO}}\right)$ and ${ }^{8} \mathrm{~B}$-neutrinos $\left(\Phi_{\mathrm{B}}\right)$ from pp-III.

We will take the following attitude:

i) we assume - as a working hypothesis - standard neutrinos (zero mass, no flavor mixing, no decay...).

ii)Through energy conservation and the assumptiom of stationary Sun, we use the observed value of the solar constant in order to constrain/determine the total neutrino flux.

iii) We rely on the SSM as least as possible (as a reference, the results of our standard solar model, hereafter the SSM, are shown in Table I). In particular, again as a working hypothesis, we assume that the SSM is unable to predict the ${ }^{8} \mathrm{~B}$-neutrino flux. This latter we take from experiment, i.e. from the Kamiokande result [4] or from the Chlorine result [5]. A choice is needed since the two experiments give almost conflicting results for standard neutrinos, see for instance refs. [6,7].

In this frame, the Gallex result [8] implies that the very large majority of neutrinos come from the pp-I termination:

$$
62 \leq \Phi_{\mathrm{p}}\left[10^{9} \mathrm{~cm}^{-2} \mathrm{~s}^{-1}\right] \leq 65,
$$

the upper bound being given by the solar luminosity constraint and the lower one essentially from the Gallex result, at $1 \sigma$. With respect to the prediction of the $\operatorname{SSM}\left(\Phi_{\mathrm{p}}{ }^{S S M}=60 \cdot 10^{9} \mathrm{~cm}^{-2} \mathrm{~s}^{-1}\right)$, this means that the fusion chain is shifted towards the pp-I termination.

Theoretically, this shift can be attained in several ways, by varying the parameters of solar models beyond the range of the observational uncertainties. One can enhance the pp-I termination by using larger (smaller) values of the ${ }^{3} \mathrm{He}+{ }^{3} \mathrm{He}\left({ }^{3} \mathrm{He}+{ }^{4} \mathrm{He}\right)$ cross section, without altering the inner solar structure (e.g. central temperature, density...). Alternatively, one can play on parameters which reduce the central temperature (opacity, age,

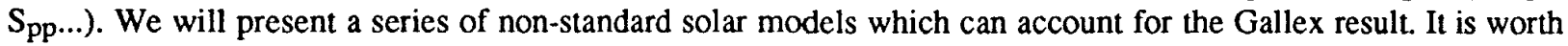
observing that all these ad-hoc models require huge variations of the parameters.

Next we investigate what can be learnt from future experiments aimed at the detection of the ${ }^{7} \mathrm{Be}$ and pep lines, in order to distinguish non-standard solar models from non-standard neutrinos.

Since the rate of fusion reactions (one half the rate of neutrino emission) is essentially fixed by solar energetics, one can derive an upper bound on the ${ }^{7} \mathrm{Be}$-neutrino flux. A measurement of the Berillium line intensity in excess of this limit will be a clear signal of non-standard neutrinos.

The pep and pp reactions are strongly connected with each other [9], so that one expects eq (1.1) can be translated into a strict prediction on $\Phi_{\text {pep }}$. In order to analyze the relevance of an experiment on pep neutrinos, 
we will compare the predictions on $\Phi_{\text {pep }}$ arising from (non-standard) solar models with those from MSW [10] oscillation models.

\section{The relevant variables and the relevant equations}

For standard neutrinos, the measurement of the solar constant $\left(\mathrm{K}=\mathrm{L}_{0} / 4 \pi \mathrm{a}^{2}=(0.849 \pm 0.002) \cdot 10^{12}\right.$ $\left.\mathrm{MeVcm}^{-2} \mathrm{sec}^{-1}\right)$ and the signals in the various neutrino experiments can be expressed as a set of linear equations, where the unknown variables are the energy integrated neutrino fluxes on Earth $\Phi_{\mathrm{i}}$. The solar constant can be written as:

$$
\mathrm{K}=\Sigma_{\mathrm{i}}\left(\mathrm{Q} / 2-\left\langle\mathrm{E}>_{\mathrm{i}}\right) \Phi_{\mathrm{i}}\right.
$$

where $Q$ is the energy released in the fusion reaction,

$$
\mathrm{Q}=4 \mathrm{~m}_{\mathrm{p}}+2 \mathrm{~m}_{\mathrm{e}}-\mathrm{m}_{\alpha}=26.73 \mathrm{MeV}
$$

and $\langle\mathrm{E}\rangle_{\mathrm{i}}$ is the average neutrino energy. Note that, if the difference among the $\langle\mathrm{E}\rangle_{\mathrm{i}}$ is neglected, eq. (2.1) actually fixes the total neutrino flux.

The signal $S_{x}$ of any experiment can be represented as:

$$
\mathrm{S}_{\mathrm{x}}=\Sigma_{\mathrm{i}} \mathrm{X}_{\mathrm{i}} \Phi_{\mathrm{i}}
$$

where the weighting factors $X_{i}$ are cross sections for the detection reaction, averaged over the spectrum of the $\mathrm{i}$ th component of the neutrino flux.

These averages do not depend on solar physics, the shape of each component of the spectrum being determined by nuclear physics alone. Note also that the $X_{i}$ are ordered according to the neutrino energy (larger $\langle E\rangle_{i}$ correspond to bigger $\mathrm{X}_{\mathrm{i}}$ ) as typical of low energy weak interaction processes, see table II for the numerical values.

As mentioned above, in order to manage the discussion, let us refer only to the main components $\Phi_{\mathrm{p}}, \Phi_{\mathrm{Be}}, \Phi_{\mathrm{B}}$ and $\Phi_{\mathrm{CNO}}=\Phi_{\mathrm{N}}+\Phi_{\mathrm{O}}$ (we neglect the very small contribution of ${ }^{17} \mathrm{~F}$-neutrinos). For the moment we take the ratio $\xi=\Phi_{\text {pep }} / \Phi_{\mathrm{p}}$ from the SSM $\left(\xi=2.38 \cdot 10^{-3}\right)$ and similarly the ratio $\eta=\Phi_{N} / \Phi_{\mathrm{CNO}}=0.54$. A reader, willing to avoid these assumptions, could take $\Phi_{\text {pep }}=0$ (actually the pep-neutrinos are a very small fraction of the pp-I) and $\eta=1 / 2$, as would be in the case of a fully operational $\mathrm{CN}$ cycle. He will get anyhow similar results.

By deriving the Boron flux from the Kamiokande result [4] ,

$$
\Phi_{\mathrm{B}} \mathrm{Ka}=(2.9 \pm 0.4) 10^{6} \mathrm{~cm}^{-2} \mathrm{~s}^{-1}
$$

( $1 \sigma$ errors here and in the following, statistical and systematical errors being added quadratically) we are left with the three unknowns $\Phi_{\mathrm{p}}, \Phi_{\mathrm{Be}}, \Phi_{\mathrm{CNO}}$, constrained by the luminosity equation,

$$
\chi=2 \mathrm{~K} / \mathrm{Q}=\alpha_{\mathrm{p}} \Phi_{\mathrm{p}}+\alpha_{\mathrm{Be}} \Phi_{\mathrm{Be}}+\alpha_{\mathrm{CNO}} \Phi_{\mathrm{CNO}}
$$

(where $\alpha_{i}=1-2<E>_{i} / Q$ and the very small contribution of ${ }^{8} B$-neutrinos is neglected) and by the Gallex result:

$$
\mathrm{S}_{\mathrm{G}}=\mathrm{S}_{\mathrm{G}}(\mathrm{B})+\mathrm{G}_{\mathrm{p}} \Phi_{\mathrm{p}}+\mathrm{G}_{\mathrm{Be}} \Phi_{\mathrm{Be}}+\mathrm{G}_{\mathrm{CNO}} \Phi_{\mathrm{CNO}}
$$


We take [8] $S_{G}=87 \pm 16 \mathrm{SNU}$ and we derive the Boron contribution to the Gallium signal, $S_{G}(B)=G_{B} \Phi_{B}$, from Kamiokande, $S_{G} \mathrm{Ka}(B)=7 \pm 2 \mathrm{SNU}$, where a $25 \%$ error $(\approx 1 \sigma)$ on $\mathrm{G}_{B}$ is assumed. The errors on the other $\mathrm{G}_{i}$ are taken to be a few percent, see table $\Pi$, and thus can be neglected in comparison with the error of the Gallex result. The factor $G_{p}$ is the average between $G_{p p}$ and $G_{p e p}\left(G_{p}=G_{p p}(1-\xi)+G_{p e p} \xi\right)$, and similarly $G_{C N O}=G_{O}(1-$ $\eta)+G_{N} \eta$. Note that we use the SSM just for evaluating $G_{p}, G_{C N O}$ and the corresponding $\alpha_{p}$ and $\alpha_{C N O}$.

Alternatively, one could derive the Boron flux from the Chlorine experiment:

$$
\mathrm{S}_{\mathrm{C}}=\mathrm{C}_{\mathrm{pep}} \Phi_{\mathrm{pep}}+\mathrm{C}_{\mathrm{Be}} \Phi_{\mathrm{Be}}+\mathrm{C}_{\mathrm{CNO}} \Phi_{\mathrm{CNO}}+\mathrm{C}_{\mathrm{B}} \Phi_{\mathrm{B}}
$$

where we take $\mathrm{S}_{C}=2.23 \pm 0.22$ [5] and the $\mathrm{C}$ coefficients are again shown in Table II. In this way the expression for the Gallex signal becomes:

$$
\mathrm{S}_{\mathrm{G}}=\mathrm{D}+\mathrm{G}_{\mathrm{p}}^{\prime} \Phi_{\mathrm{p}}+\mathrm{G}_{\mathrm{Be}}^{\prime} \Phi_{\mathrm{Be}}+\mathrm{G}_{\mathrm{CNO}}^{\prime} \Phi_{\mathrm{CNO}}
$$

where $D=S_{C}\left(G_{B} / C_{B}\right)$ and $G_{B e}^{\prime}=G_{B e}-C_{B e}\left(G_{B} / C_{B}\right)$, similarly for $G_{C N O}^{\prime}$, whereas $G_{p}^{\prime}=G_{p}-\xi C_{p}\left(G_{B} / C_{B}\right)$.

Eqs. (2.4) and (2.5), or alternatively (2.7), are at the basis of our discussion.

\section{The solar luminosity, the Gallex result and the flux of pp-I neutrinos}

The solar luminosity equation (2.4) provides a lower limit to the total neutrino flux, which is reached when all neutrinos are from pp-I :

$$
\Phi^{\text {tot }} \geq \chi / \alpha_{\mathrm{p}}=64.8 \cdot 10^{9} \mathrm{~cm}^{-2} \mathrm{~s}^{-1}
$$

This is due to the fact that $\mathrm{pp}-\mathrm{I}$ neutrinos are the least energetic ones. Clearly, it also provides an upper bound to the flux of pp-I neutrinos, $\Phi_{\mathrm{p}} \leq \Phi_{\mathrm{p}} \max =\chi / \alpha_{\mathrm{p}}$.

When used in conjunction with the Gallex result, the luminosity equation gives useful information on the way fusion reactions proceed in the Sun. For example, the CNO cycle, as the main source of solar energy $[11,12]$, can immediately be rejected. The luminosity would require $\Phi_{\mathrm{CNO}}=67.8 \cdot 10^{9} \mathrm{~cm}^{-2} \mathrm{~s}^{-1}$, whereas from eq. (2.5) one immediately gets $\Phi_{\mathrm{CNO}} \leq 11 \cdot 10^{9} \mathrm{~cm}^{-2} \mathrm{~s}^{-1}$ (more stringent bounds can be derived from the following discussion).

In order to gain information about the pp-I neutrinos, we observe that the minimum signal in the Gallex experiment is obtained when all neutrinos (but the small ${ }^{8} \mathrm{~B}$ fraction) are from $\mathrm{pp}-\mathrm{I}$, since in this case $\Phi$ tot is minimized and all neutrinos have the lowest energy. By adding the small contribution of ${ }^{8} \mathrm{~B}$-neutrinos:

$$
\mathrm{S}_{\mathrm{G}} \geq \mathrm{G}_{\mathrm{p}} \chi / \alpha_{\mathrm{p}}+\mathrm{S}_{\mathrm{G}} \mathrm{Ka}(\mathrm{B})=86.6 \mathrm{SNU} \text {. }
$$

This value being very close to the experimental result, one understands that pp-I neutrinos must be a substantial component of the total flux. For a quantitative determination of this component, let us see what happens when other neutrinos ( ${ }^{7} \mathrm{Be}$ and CNO) are present, i.e. for $\Phi_{\mathrm{p}}<\Phi_{\mathrm{p}} \max$. For a given value of $\Phi_{\mathrm{p}}$ the minimum signal is obtained when all other neutrinos are from Berillium, so that the energy is as low as possible. This gives an expression of the minimum signal for Gallex as a function of $\Phi_{\mathrm{p}}$ :

$$
\mathrm{S}_{G^{m i n}}\left(\Phi_{\mathrm{p}}\right)=\mathrm{S}_{\mathrm{G}} \mathrm{Ka}(\mathrm{B})+\mathrm{G}_{\mathrm{Be}} \chi / \alpha_{\mathrm{Be}}+\left(\mathrm{G}_{\mathrm{p}}-\mathrm{G}_{\mathrm{Be}} \alpha_{\mathrm{p}} / \alpha_{\mathrm{Be}}\right) \Phi_{\mathrm{p}}
$$

In a similar way, the maximum signal $\mathrm{S}_{\mathrm{Ga}}{ }^{\max }\left(\Phi_{\mathrm{p}}\right)$ is obtained when all other neutrinos are from the CNO cycle: 


$$
\mathrm{S}_{\mathrm{G}} \max \left(\Phi_{\mathrm{p}}\right)=\mathrm{S}_{\mathrm{G}} \mathrm{Ka}(\mathrm{B})+\mathrm{G}_{\mathrm{CNO}} \chi / \alpha_{\mathrm{CNO} O}+\left(\mathrm{G}_{\mathrm{p}}-\mathrm{G}_{\mathrm{CNO}} \alpha_{\mathrm{p}} / \alpha_{\mathrm{CNO}}\right) \Phi_{\mathrm{p}}
$$

The allowed region for conventional neutrinos is thus the small area within the two straight lines, $\mathbf{S}_{\mathrm{G}} \min \left(\Phi_{\mathrm{p}}\right)$ and $\mathrm{S}_{\mathrm{G}} \max \left(\Phi_{\mathrm{p}}\right)$, see Fig. 1. Taking into account the experimental value of $\mathrm{S}_{\mathrm{G}}$, one finds:

$$
62.3 \leq \Phi_{\mathrm{p}}\left[10^{9} \mathrm{~cm}^{-2} \mathrm{~s}^{-1}\right] \leq 64.8
$$

where the upper bound is given by the luminosity constraint and the lower one from Gallex result, at $1 \sigma$.

By using the Chlorine result instead of Kamiokande, one gets a similar conclusion:

$$
61.6 \leq \Phi_{\mathrm{p}}\left[10^{9} \mathrm{~cm}^{-2} \mathrm{~s}^{-1}\right] \leq 64.8 \text {. }
$$

It is worth observing that Gallex experiment, in spite of its error of about $20 \%$, fixes the pp-I neutrino flux within 5\%. The point is that neutrinos different from pp-I have a large weighting factor in the Gallium signal, so that even a small flux gives an appreciable contribution.

The result has to be compared with the SSM value, $\Phi_{\mathrm{p}} \mathrm{SSM}=60 \cdot 10^{9} \mathrm{~cm}^{-2} \mathrm{~s}^{-1}$, affected by an uncertainty $(\approx 1 \sigma)$ of about $0.7 \%$, see ref. [13]. Although the SSM value may seem not too far from the above bounds, actually solar models which can account for the experimental value of $\Phi_{p}$ - eq. (1.1) - are extremely non-standard, in the sense that huge variations of the relevant parameters, well beyond the range of uncertainties, are to be introduced (for results of solar models close to the SSM see ref.[14]).

Note also that bringing $\Phi_{\mathrm{p}}$ into the right range is a necessary condition but it is not sufficient to fully account for the Gallex result. In fact, even if $\Phi_{p}$ is right, there is generally a contribution from CNO-neutrinos, so that the signal can be larger than the minimum value given by eq. (3.3). The results of several models for the Gallium signal (we remind that the Boron contribution is anyhow directly taken from the Kamiokande experiment) are shown in Fig. 2, as some solar model parameter $\mathrm{x}$ is varied from its standard value $\mathrm{x}_{\mathbf{S S M}}$.

In order to enhance the pp-I branch of the fusion reaction chain we resorted to several manipulations:

i) a large ${ }^{3} \mathrm{He}+3 \mathrm{He}$ cross section at low energy. This nuclear physics solution requires an astrophysical S-factor larger than the standard value by a factor at least fifteen, see Fig. 2 (we require that the Gallex value is reproduced at $1 \sigma$ level). The possibility of a low energy resonance cannot be excluded $[7,15,16]$ and is presently investigated experimentally [17]. Note that the internal solar structure is minimally changed, the central solar temperature $T_{c}$ being reduced by just $5 \%$ with respect to $T_{c}$ SSM for $S_{33} / S_{33} S S M=50$. Alternatively one could diminish the cross section for the ${ }^{3} \mathrm{He}+{ }^{4} \mathrm{He}$ reaction, getting similar conclusions since all neutrino fluxes depend just on the ratio $S_{33} / S_{34}{ }^{2}$, see ref. [7].

ii) A large p-p cross section. Increasing $S_{p p}$ implies a lower central temperature, since fusion gets easier while the solar luminosity has to be kept constant [18]. Clearly at lower temperatures the pp-II termination and the CNO cycle are disfavoured. Although $S_{\mathrm{pp}}$ is well determined, one can introduce an artificial variation just in order to get cooler Suns. One needs $S_{\mathrm{pp}}$ at least twice the standard value, corresponding to a central temperature $\mathrm{T}_{c} \leq 1.4410^{7} \mathrm{oK}$, about $8 \%$ smaller than in the SSM.

iii) A less opaque Sun is another way to get a cooler solar interior. This can be accomplished by lowering (by an overall multiplicative factor $\mathrm{x}_{\mathrm{opa}}$ ) the opacity tables or by decreasing the metal fraction $\mathrm{Z} / \mathrm{X}$. We have lowered the opacity tables down to $\mathrm{x}_{\mathrm{opa}}=0.6$. We did not proceed any further because otherwise we cannot produce stable solar models. Also for $x_{o p a}=0.6$ the calculated Gallium signal exceeds the experimental result. The value of $\mathrm{Z} / \mathrm{X}$ needed to reproduce the Gallex result at $1 \sigma$ is $\mathrm{Z} / \mathrm{X} \leq 0.3 \cdot(\mathrm{Z} / \mathrm{X})_{S S M}$, the central temperature being decreased by about $6 \%$. 
iv) A younger Sun is another way to get a cooler Sun. Agreement with Gallex cannot be reached even for a Sun $5 \cdot 10^{8}$ y old!

Note that the common point of models ii), iii) and iv) is the reduction of the central temperature. Different models give similar results for the Gallium signal when the central temperature is the same, see Fig. 3.

Clearly all these models are desperate attempts in order to save the hypothesis of standard neutrinos. We will discuss in the next section how future experiments can distinguish non-standard solar models from non- standard neutrinos.

\section{4. ${ }^{7} \mathrm{Be}$ and pep-neutrinos}

If the fusion chain is shifted towards the pp-I termination, as shown in the previous section, the flux of ${ }^{7} \mathrm{Be}$ neutrinos has to be reduced with respect to the SSM, since the total neutrino flux is fixed by the solar luminosity. We recall that the minimum Gallium signal occurs when the CNO flux is put equal to zero. By expressing $\Phi_{\mathrm{p}}$ in terms of $\Phi_{\mathrm{Be}}$ through the luminosity constraint, eq. (3.3) becomes:

$$
S_{G} \min \left(\Phi_{B e}\right)=S_{G} K a(B)+G_{p} \chi / \alpha_{p}+\left(G_{B e}-G_{p} \alpha_{B e} / \alpha_{p}\right) \Phi_{B e}
$$

In Fig. 4 the region of non-standard neutrinos lies below the full line. Assuming standard neutrinos, the Gallex result at $1 \sigma$ then implies:

$$
\Phi_{\mathrm{Be}} \leq 2.7 \cdot 10^{9} \mathrm{~cm}^{-2} \mathrm{~s}^{-1}
$$

If the Chlorine result is used, one gets $\Phi_{\mathrm{Be}} \leq 3.3 \cdot 10^{9} \mathrm{~cm}^{-2} \mathrm{~s}^{-1}$. The skeptical reader of sect. 2 would get $\Phi_{\mathrm{Be}} \leq$ $3.1 \cdot 10^{9} \mathrm{~cm}^{-2} \mathrm{~s}^{-1}$ instead of (4.2), and by using the Chlorine results $\Phi_{\mathrm{Be}} \leq 3.8 \cdot 10^{9} \mathrm{~cm}^{-2} \mathrm{~s}^{-1}$. Note that these limiting values are definitely smaller than $\Phi_{\mathrm{Be}} \mathrm{SSM}=4.8 \cdot 10^{9} \mathrm{~cm}^{-2} \mathrm{~s}^{-1}$.

A measurement of $\Phi_{\mathrm{Be}}$ in excess of these bounds would be a clear signal of non-standard neutrinos.

It has to be observed that the MSW solution of the solar neutrino problem does not put any significant constraint on $\Phi_{\mathrm{Be}}$, the allowed range being $(1.1 \div 3.6) \cdot 10^{9} \mathrm{~cm}^{-2} \mathrm{~s}^{-1}$ at $90 \%$ C.L.[19].

One can repeat the same discussion in order to obtain an upper bound for the CNO flux (in this case $\Phi_{\mathrm{Be}}$ is taken equal to zero). One finds $\Phi_{\mathrm{CNO}} \leq 2.2 \cdot 10^{9} \mathrm{~cm}^{-2} \mathrm{~s}^{-1}$ if the Boron flux is taken from Kamiokande and $\Phi_{\mathrm{CNO}} \leq$ $2.9 \cdot 10^{9} \mathrm{~cm}^{-2} \mathrm{~s}^{-1}$ if one derives $\Phi_{\mathrm{B}}$ from the Chlorine experiment. This means that in any case the CNO cycle does not contribute more than $5 \%$ to the solar energy production.

Pep neutrinos look very interesting since that the pep and pp reactions are strongly connected, the pep-rate being proportional to the pp one $\left(\lambda_{\text {pep }} \propto n_{e} \lambda_{p p}\right.$, where $\lambda_{i}$ are the rates and $n_{e}$ is the electron density). One thus expects that the ratio $\xi=\Phi_{\text {pep }} / \Phi_{\mathrm{p}}$ is rather stable as solar models are varied, so that a measurement of the pep line intensity is a direct test of the pp-I termination of the fusion chain.

In the SSM one has $\xi=2.3810^{-3}$, with an estimated error of $0.8 \%$ at $1 \sigma$ level, essentially due to the uncertainties on Spp and on the metal fraction $\mathrm{Z} / \mathrm{X}$ [13]. From the constraints on $\Phi_{\mathrm{p}}$ found in the previous section, we derive that $\Phi_{\text {pep }}$ must lie in a very narrow range:

$$
0.147 \leq \Phi_{\text {pep }}\left[10^{9} \mathrm{~cm}^{-2} \mathrm{~s}^{-1}\right] \leq 0.154
$$

Bahcall [13] has shown that the factor $\xi$ is rather stable against moderate variations around the SSM. Actually, in order to save standard neutrinos one must resort to very non-standard model, where the value of $\xi$ may be somehow different. We have investigated the prediction of $\Phi_{\text {pep }}$ in a large class of non-standard model adjusted 
so as to reproduce the Gallex result within $1 \sigma$ (the Boron contribution anyhow being taken from the Kamiokande experiment). We find that $\Phi_{\text {pep }}$ stays within $(0.1 \div 0.2) \cdot 10^{9} \mathrm{~cm}^{-2} \mathrm{~s}^{-1}$ even for huge variations of the parameters, see Fig. 5.

It is interesting to compare this range with that allowed for the MSW (non-adiabatic) solution at $90 \%$ C.L., see again Fig. 5 and for more details Fig. 6 and ref. [19] :

$$
\Phi_{\text {pep }}{ }^{M S W} \leq 3 \cdot 10^{7} \mathrm{~cm}^{-2} \mathrm{~s}^{-1}
$$

Note that a very low pep-neutrino flux is mandatory for MSW, since the Chlorine and Kamiokande result can be recoinciled by the MSW effect when the low energy part of the Boron spectrum is strongly suppressed, and this overlies the pep energy. The same is generally true for any mechanism (resonant spin flip, neutrino decay...) which attemps to reconcile Chlorine and Kamiokande results.

In conclusion, the measurement of the pep line intensity will clearly distinguish non-standard solar models from non-standard neutrinos (as recently pointed out in ref.[3]). Futhermore, as shown in Fig. 5, the measurement of $\Phi_{\text {pep }}$ can also discriminate among different solar models.

\section{Conclusions}

We summarize the main points of our discussion:

i) The Gallex result together with the luminosity constraint implies that almost all neutrinos, if standard, are from the pp-I termination

$$
62 \leq \Phi_{\mathrm{pp}+\mathrm{pep}}\left[10^{9} \mathrm{~cm}^{-2} \mathrm{~s}^{-1}\right] \leq 65 .
$$

The strict bound derives from the fact that even a small flux of other (=more energetic) neutrinos gives an appreciable contribution to the Gallium signal.

ii) A clear check of neutrino properties can be obtained by looking at ${ }^{7} \mathrm{Be}$-neutrinos: $\Phi_{\mathrm{Be}} \leq 3.10^{9} \mathrm{~cm}^{-2} \mathrm{~s}^{-1}$ or neutrinos are non-standard;

iii) (non-)standard solar models give always a large pep flux, $\Phi_{\text {pep }} \approx 1.5 \cdot 10^{8} \mathrm{~cm}^{-2} \mathrm{~s}^{-1}$, whereas the MSW solution requires $\Phi_{\text {pep }}{ }^{M S W} \leq 3 \cdot 10^{7} \mathrm{~cm}^{-2} \mathrm{~s}^{-1}$ (and even a smaller value for the best fit). The measurement of the pep line intensity will clearly distinguish non-standard solar models from non-standard neutrinos. Futhermore, it can also discriminate among different solar models.

\section{Acknowledgements}

It is a pleasure to thank E. Fiorini, G. Mezzorani, M. Moretti and D. Vignaud for fruitful discussions. 


\section{References}

[1] C. Arpesella et al, Borexino at Gran Sasso: Proposal for a Real-Time Detector for Low Energy Solar Neutrinos, internal report INFN - Milan (1992).

[2] A. Alessandrello et al., A Cryogenic Experiment for Solar Neutrino Spectroscopy and Search for Dark Matter, preprint INFN/AE-92/28 (1992).

[3] R. S. Raghavan et al, High Resolution Spectroscopy of Solar Neutrino by Neutral and Charged Currents

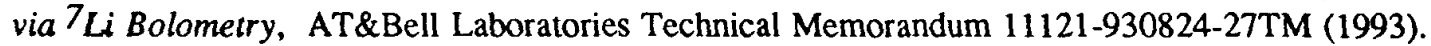

[4] A. Suzuki, Solar Neutrinos Observation in Kamiokande, in Neutrino Telescopes '93, Ed. M.B. Ceolin, Venice (1993).

[5] E. Lande, talk given at Particle Astrophysics, Chateau Blois, France, 15-20 June 1992, to appear in the proceedings of the conference.

[6] J.N. Bahcall and H.A. Bethe, Phys. Rev. D44 (1991) 2962.

[7] V. Castellani et al., A. \& A. 271 (1993) 601.

[8] GALLEX Collaboration, Phys. Lett. B314 (1993) 443.

[9] J.N. Bahcall and R.M. May, Ap. J. 155 (1969) 501.

[10] S.P. Mikheyev and A.Y. Smirnov, Nuovo Cimento 9C (1986) 17;

L.Wolfenstein, Phys. Rev. D17 (1978) 2639.

[11] H.A. Bethe, Phys. Rev. 55 (1939), 103.

[12] C. F. Weizsaecker, Physik. Zeits. 39 (1938) 639.

[13] J.N. Bahcall, Neutrino Astrophysics, Cambridge University Press, Cambridge (1989).

[14] J.N. Bahcall and H.A. Bethe, Phys. Rev. D47 (1993) 1298.

[15] W. A. Fowler, Nature 238 (1972) 24

[16] A. Krauss et al., Nucl. Phys. A467 (1987) 273.

[17] C. Arpesella et al, Nuclear Astrophysics at Gran Sasso Laboratory (Proposal for a pilot project with a 30 $\mathrm{KeV}$ accelerator) internal report LNGS 91-18 (1991).

[18] V. Castellani et al., Phys. Lett. B303 (1993) 68

[19] G. Fiorentini et al., Solar Neutrino Experiments and Determination of the Neutrino Oscillation Parameters, preprint INFN-FE-10-93 (1993).

[20] A. Garcia et al., Phys. Rev. Lett. 67 (1991) 3654.

Table I

Our Standard Solar Model. We show the age, the central temperature $\left(T_{c}\right)$, the Helium mass fraction $(Y)$, the metal mass fraction (Z) and the neutrino fluxes, in units of $10^{9} \mathrm{~cm}^{-2} \mathrm{~s}^{-1}$. Also shown are the theoretical values for the Chlorine and Gallium signals.

\begin{tabular}{|l|c|}
\hline Age [Gyr] & 4.6 \\
\hline$T_{\mathrm{C}}\left[{ }^{0} \mathrm{~K}\right]$ & $1.564 \cdot 10^{7}$ \\
\hline $\mathrm{Y}$ & 0.289 \\
\hline $\mathrm{Z}$ & 0.0184 \\
\hline$\Phi_{\mathrm{pp}}$ & 60.05 \\
\hline$\Phi_{\mathrm{pep}}$ & 0.14 \\
\hline$\Phi_{\mathrm{D}}=\Phi_{\mathrm{pD}}+\Phi_{\mathrm{pep}}$ & 60.19 \\
\hline$\Phi_{\mathrm{Be}}$ & 4.79 \\
\hline$\Phi_{\mathrm{N}}$ & 0.47 \\
\hline$\Phi_{\mathrm{Q}}$ & 0.40 \\
\hline$\Phi_{\mathrm{CNO}}=\Phi_{\mathrm{N}}+\Phi_{\mathrm{O}}$ & 0.87 \\
\hline$\Phi_{\mathrm{B}}$ & $5.6 \cdot 10^{-3}$ \\
\hline Chlorine $[\mathrm{SNU}]$ & 7.8 \\
\hline Gallium $[\mathrm{SNU}]$ & 130 \\
\hline
\end{tabular}




\section{Table II}

For the different components of the neutrino flux we show the average neutrino energy $\langle E\rangle$ and the averaged neutrino capture cross sections for Chiorine (C) and Gallium (G), with errors at $1 \sigma$ level, from ref. [13] but for $C_{B}$ taken from ref. [20]. When averaging the pp and pep components we use the relative weights of our SSM, see Table I; similarly for ${ }^{13} \mathrm{~N}$ and ${ }^{15} \mathrm{O}$.

\begin{tabular}{|l|c|c|c|}
\hline & $\begin{array}{c}\text { <E> } \\
{[\mathrm{MeV}]}\end{array}$ & $\begin{array}{c}\mathrm{C} \\
{\left[10^{9} \mathrm{SNUcm}^{2} \mathrm{~s}\right]}\end{array}$ & $\left.\begin{array}{c}\mathrm{G} \\
{\left[10^{-9} \mathrm{SNUcm}\right.}\end{array}{ }^{2} \mathrm{~s}\right]$ \\
\hline $\mathrm{pp}$ & 0.265 & 0 & $1.18(1 \pm 0.02)$ \\
\hline $\mathrm{pep}$ & 1.442 & $1.6(1 \pm 0.02)$ & $21.5(1 \pm 0.07)$ \\
\hline $\mathrm{p}=\mathrm{pp}+\mathrm{pep}$ & 0.268 & & $1.23(1 \pm 0.02)$ \\
\hline${ }^{7 \mathrm{Be}}$ & 0.814 & $0.24(1 \pm 0.02)$ & $7.32(1 \pm 0.03)$ \\
\hline${ }^{13} \mathrm{~N}$ & 0.707 & $0.17(1 \pm 0.02)$ & $6.18(1 \pm 0.03)$ \\
\hline $15 \mathrm{O}$ & 0.996 & $0.68(1 \pm 0.02)$ & $11.6(1 \pm 0.06)$ \\
\hline $\mathrm{CNO}={ }^{13} \mathrm{~N}+15 \mathrm{O}$ & 0.840 & $0.40(1 \pm 0.02)$ & $8.67(1 \pm 0.05)$ \\
\hline${ }^{8} \mathrm{~B}$ & 6.71 & $1.09 \cdot 10^{3}(1 \pm 0.01)$ & $2.43 \cdot 10^{3}(1 \pm 0.25)$ \\
\hline
\end{tabular}

\section{Figure captions}

Fig.1. The Gallium signal $S_{G}$ as a function of the neutrino flux $\Phi_{p}$ from the pp-I termination. The Gallex result $\pm 1 \sigma$ is shown. For standard neutrinos, the allowed area is within the two straight lines $S_{G^{m i n}}$ and $S_{G^{m a x}}$ (we derived the Boron contribution from the Kamiokande experiment). The region consistent with the Gallex result and standard neutrinos is the shaded area. The lower limit for the pp-I flux is thus $\Phi_{\mathrm{p}^{m i n}}=62.3 \cdot 10^{9} \mathrm{~cm}^{-2} \mathrm{~s}^{-1}$ and the upper limit, given by the luminosity constraint, is $\Phi_{\mathrm{p}} \max =64.8 \cdot 10^{9} \mathrm{~cm}^{-2} \mathrm{~s}^{-1}$. The upper scale represents the pep-neutrino flux $\Phi_{\text {pep }}$, for a ratio $\xi=\Phi_{\mathrm{pep}} / \Phi_{\mathrm{p}}=2.38 \cdot 10^{-3}$ as in the SSM. For reference, the result of our SSM is also shown $(\diamond)$.

Fig. 2. The Gallium $S_{G}$ signal given by various non-standard solar models when some parameter $x$ is varied from the standard value $x_{S S M}$ : we consider variations of $S_{33}(X), S_{p p}(\square)$, opacity $(\not), Z X X(\Psi)$ and age $(\downarrow)$. The region consistent with the Gallex result at $1 \sigma$ level lies within the dashed lines. The Boron contribution is taken from the Kamiokande experiment for the standard model ("SSM") as well as for the other models.

Fig. 3. The Gallium signal as a function of central solar temperature $T_{c}$, in units of $T_{c} S S M$, same notation as in Fig. 2.

Fig. 4. The Gallium signal $S_{\mathrm{G}}$ as a function of the ${ }^{7} \mathrm{Be}$-neutrino flux $\Phi_{\mathrm{Be}}$. The Gallex result $\pm 1 \sigma$ is also shown (dashed lines). For standard neutrinos, the allowed area is above the straight line $S_{\mathrm{G}} \min \left(\Phi_{\mathrm{Be}}\right)$, if the Boron contribution is derived from the Kamiokande experiment. The region consistent with the Gallex result and standard neutrinos is the shaded area. The allowed flux has to be smaller than $\Phi_{B e^{m a x}}=2.7 \cdot 10^{9} \mathrm{~cm}^{-2} \mathrm{~s}^{-1}$. The result of our SSM is also shown $(\diamond)$.

Fig. 5. The pep-neutrino flux $\left(\Phi_{\text {pep }}\right)$ vs. the ${ }^{7}$ Be-neutrino one $\left(\Phi_{B e}\right)$ :

-for the standard solar model $(0)$.

-For several non standard solar models adjusted so as to reproduce the Gallex result within $1 \sigma$ (the Boron contribution anyhow being taken form Kamiokande experiment). Same notation as in Fig.2. The numbers close to each point represent the corresponding value of $\mathrm{x} / \mathrm{xSSM}$.

-The values for the MSW solution, for the best fit $(X)$ and for the $90 \%$ C.L. region (dots), see also ref [16].

Fig. 6. Iso-signal curves in the MSW model for the pep line, normalized to the SSM value. Labels refer to solid curves and the dashed curves are half-way the solid ones. The $90 \%$ C.L. region (long-dashed curves) and the best fit to the present experimental data (Chlorine, Kamiokande and Gallex) are also shown ( () ). The best fit [19] is obtained for $\Delta \mathrm{m}^{2}=6.9 \cdot 10^{-6} \mathrm{eV}{ }^{2}, \sin ^{2} 2 \theta=5.8 \cdot 10^{-3}$ and it corresponds to $\Phi_{\text {pep }} / \Phi_{\text {pep }} S S M=3.5 \%$. 


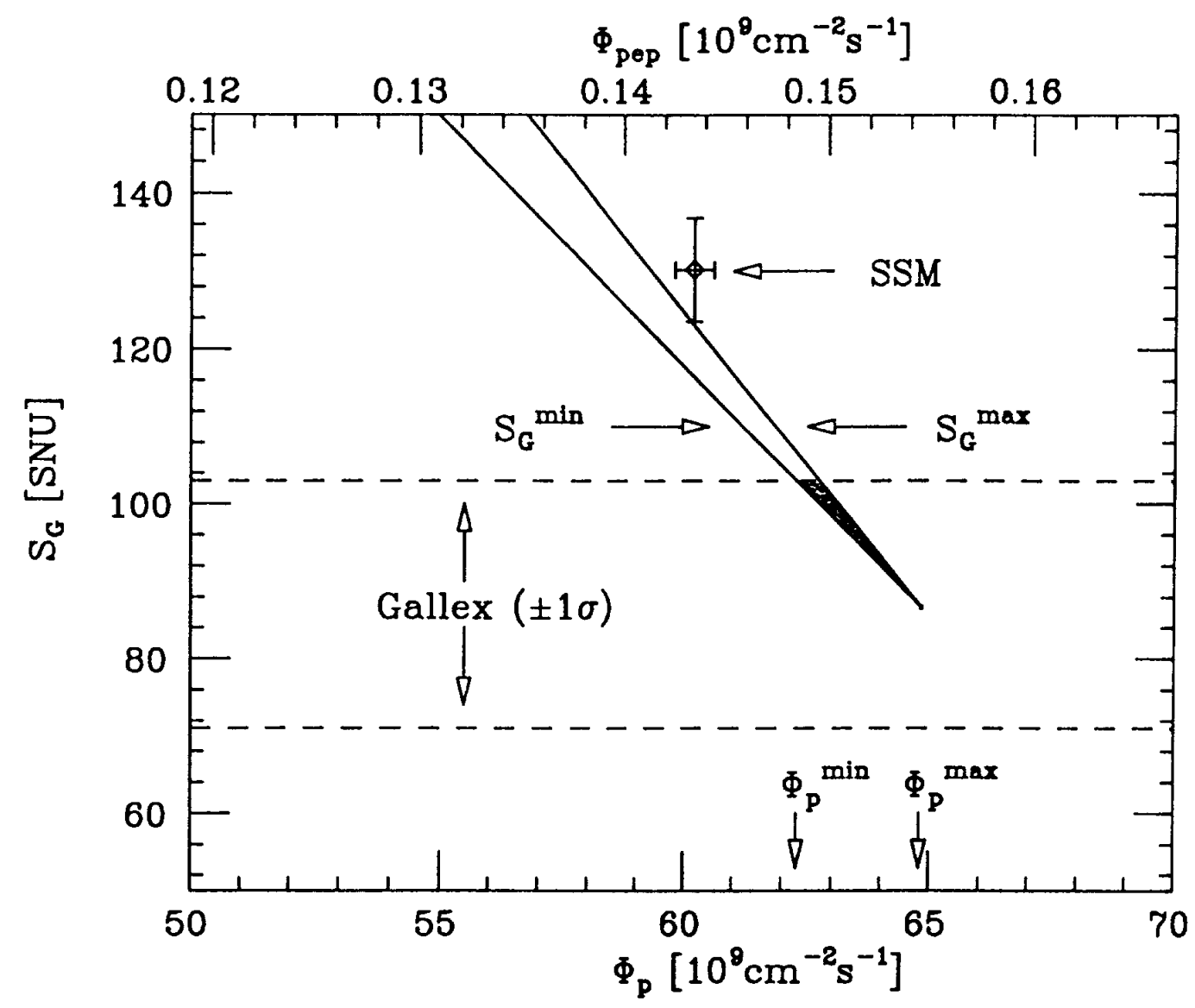

Fig. 1

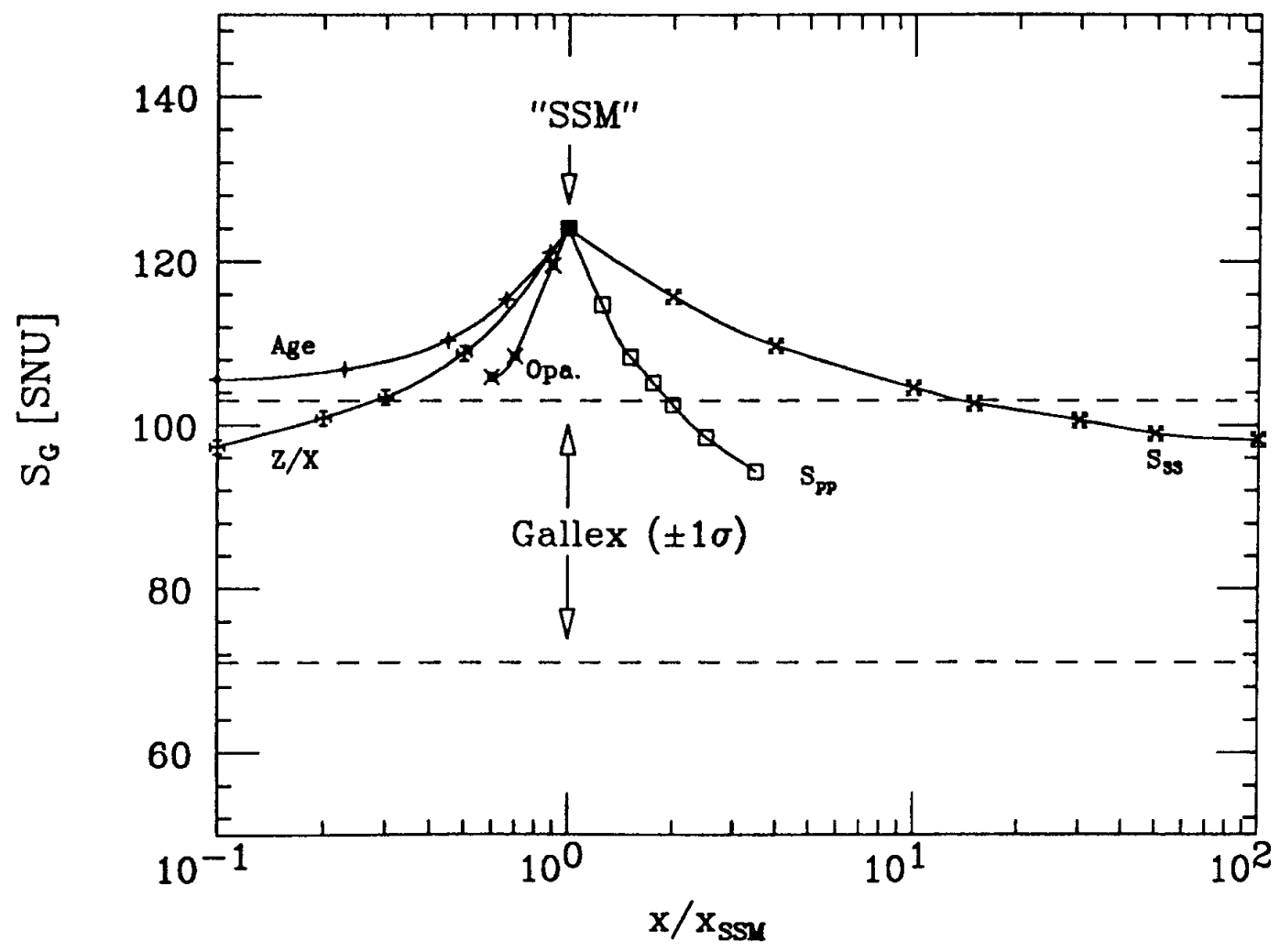

Fig. 2 


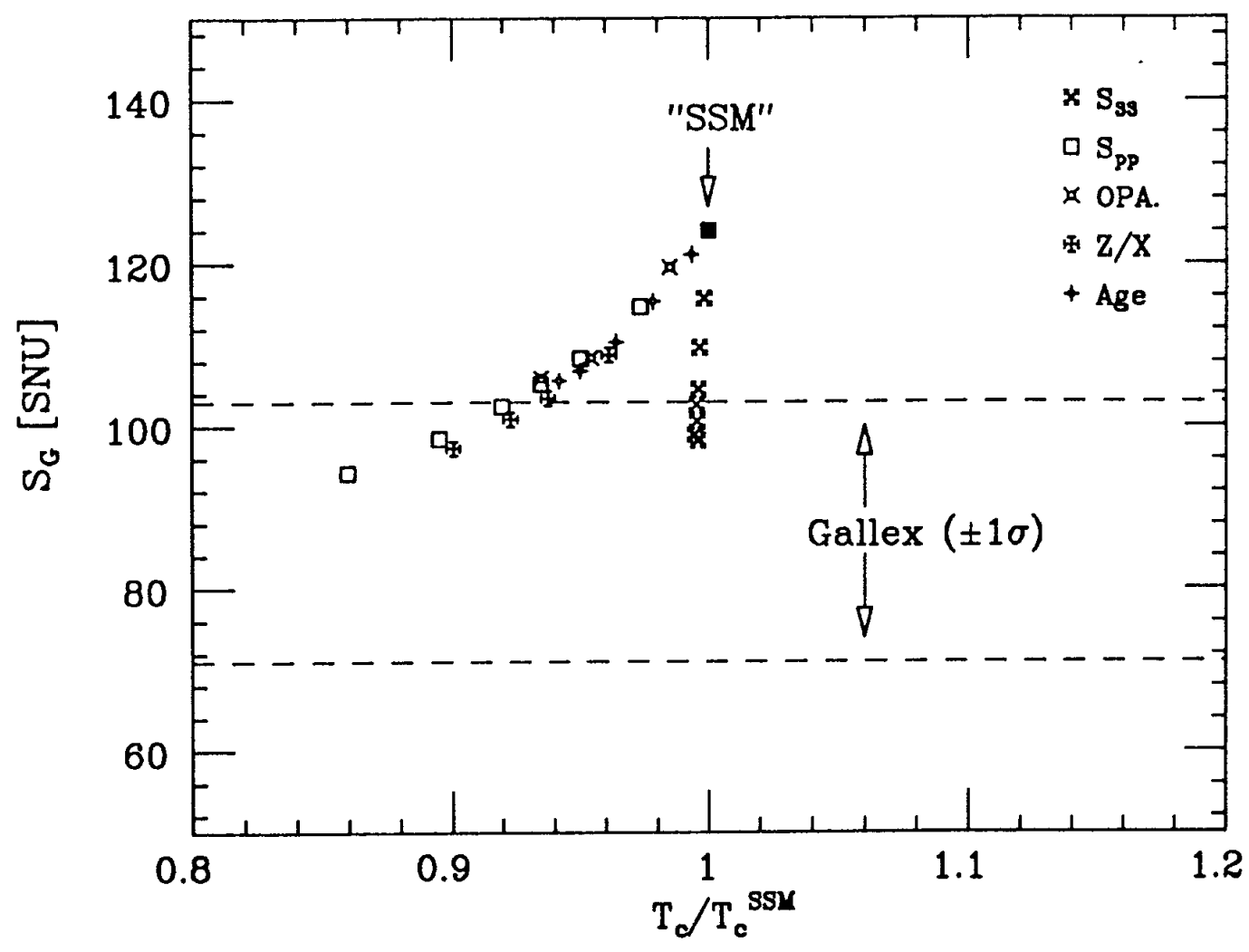

Fig. 3

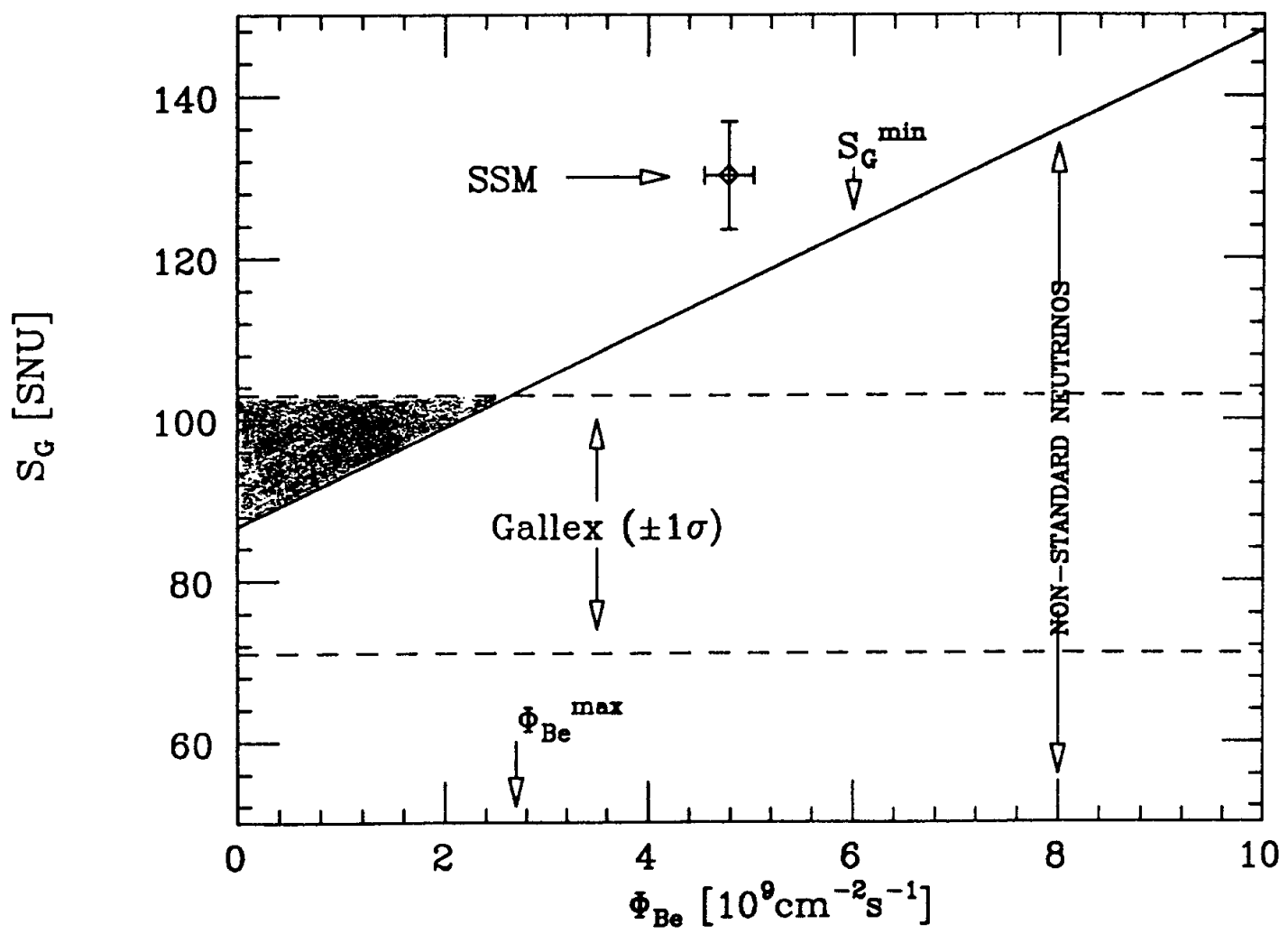

Fig. 4 


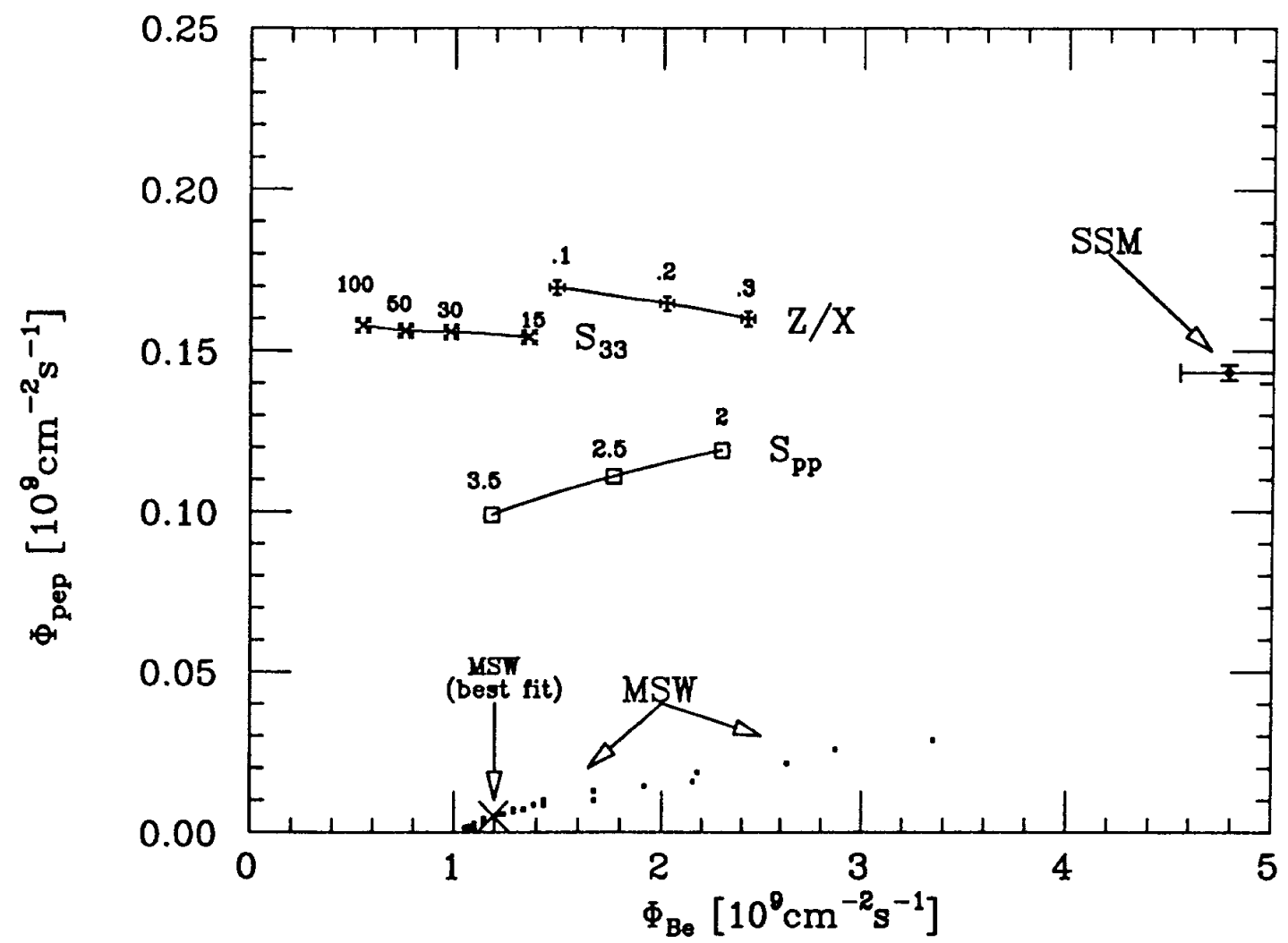

Fig. 5

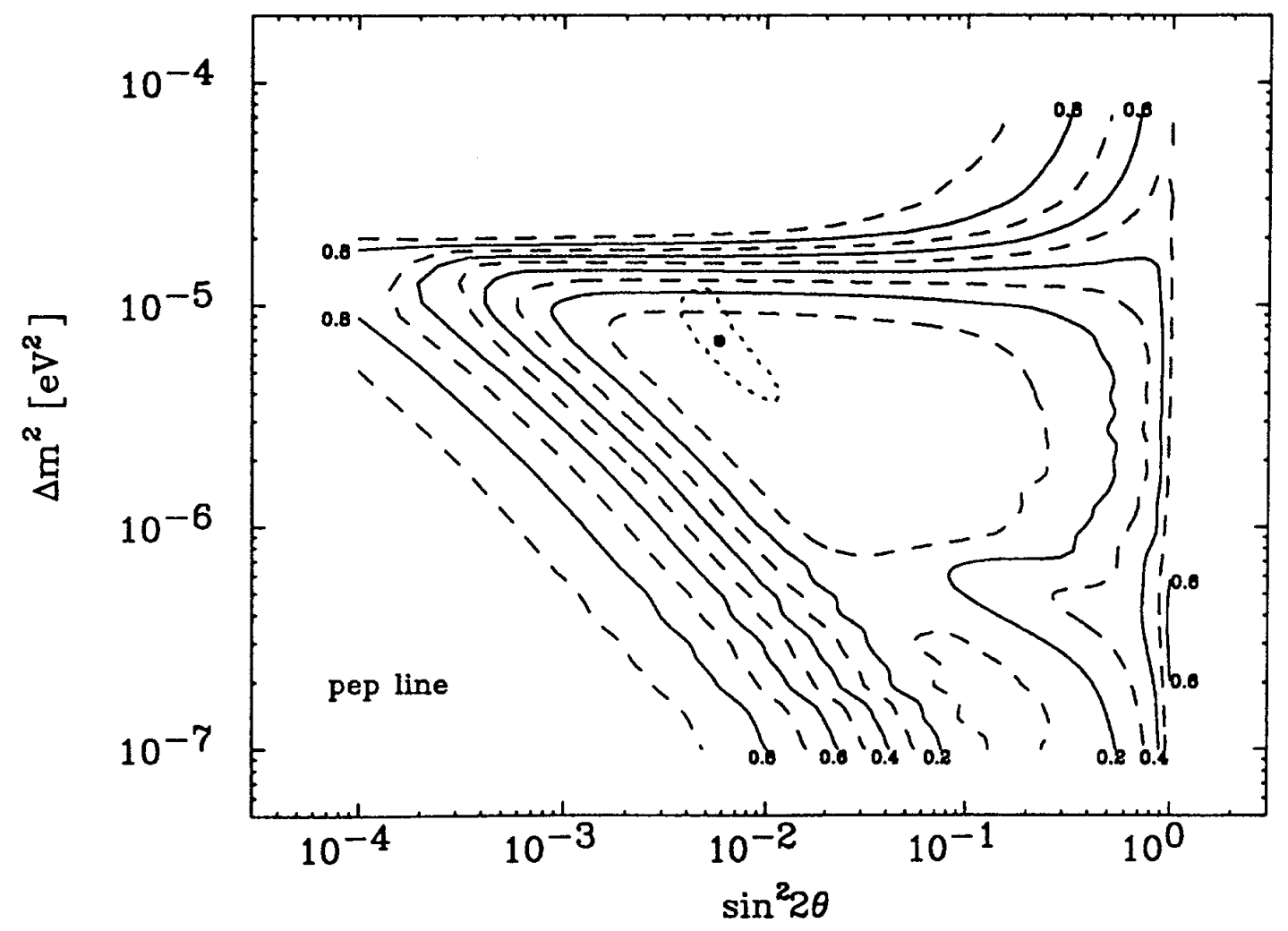

Fig. 6 